\title{
Comparaison structurale des acides humiques et du concentré de kérogène du phosphate de Youssoufia riche en matière organique
}

\author{
Mohmed KHADDOR ${ }^{*}$ et Mahfoud ZIYAD ${ }^{2}$ \\ 'Laboratoire Physico-Chimie des matériaux, Substances Naturelles et Environnement, Faculté \\ des Sciences et Techniques, Tanger, Maroc. \\ 2Laboratoire de Physico-Chimie des Matériaux et Catalyse, Faculté des Sciences, Rabat, Maroc.
}

*Correspondance,courriel : mkhaddor@hotmail.com

\section{Résumé}

De nombreux travaux ont été développés sur l'étude de la matière organique contenue dans le phosphate naturel de Youssoufia. La compréhension des mécanismes réactionnels engendrés lors de la calcination, permet d'avoir des renseignements importants sur la structure de la matière organique.

Dans un premier temps, nous avons essayé d'extraire les acides humiques et le concentré de kérogène du phosphate noir de Youssoufia. La R.M.N ${ }^{13} \mathrm{C}$ du solide, I'DSC et I'A.T.G ont permis d'identifier les principaux constituants de la matière organique des acides humiques et du concentré de kérogène.

Nous avons également étudié la cinétique de dégagement des gaz émanants des acides humiques et $d u$ concentré de kérogène, extraits de la matière organique. Nous nous sommes intéressés principalement aux dégagements du $\mathrm{CO}_{2}, \mathrm{H}_{2}$ et $\mathrm{CH}_{4}$ :

- Le $\mathrm{CO}_{2}$ semble avoir presque la même allure pour le concentré de kérogène et les acides humiques. La position des maxima des pics de production de $\mathrm{CO}_{2}$ est la même pour les deux échantillons.

- L'hydrogène et le $\mathrm{CH}_{4}$ se dégagent des acides humiques et du concentré de kérogène en une seule vague.

Mots-clés : Phosphate, matière organique, IR-FT, $R M N{ }^{13} \mathrm{C} d u$ solide pyrolyse 


\section{Abstract \\ Structural comparison of humic acids and of kerogen concentration from youssoufia phosphate rich in organic matter}

Many works have been developed on the study of the organic matter from the Moroccan Youssoufia rock phosphate. The understanding of the mechanisms generated from that time of the calcination, permits to have important information of the organic matter structure.

In a first time, we tried to extract the humic acids and the kerogen concentrate of the black Youssoufia phosphate.

The solid state ${ }^{13} \mathrm{C}$ NMR, the DSC and the A.T.G permitted identification the constituent principals of the organic matter from the humic acids and from the kerogen concentrate.

We also studied the kinetics of the release gases emanating from the humic acids and from the kerogen concentrate.We were interested mainly in the release of the $\mathrm{CO}_{2}, \mathrm{H}_{2}$ and $\mathrm{CH}_{4}$ :

- The $\mathrm{CO}_{2}$ seems to have the same pace nearly for kerogen concentrate and the humic acids. The position of the maximum release of $\mathrm{CO}_{2}$ is the same for the two samples.

- Hydrogen and methane release from the humic acids and the kerogen concentrate with only one wave.

Keywords : Phosphate, organic matter, FT-IR, solid ${ }^{13}$ C NMR, pyrolysis

\section{Introduction}

La matière organique est un constituant ubiquiste des roches sédimentaires. Dans certains sédimentaires anciens, les teneurs peuvent être relativement élevées. Les géochimistes attribuent l'existence de cette matière organique à des accumulations de molécules fossiles d'origine biologique, issues des organismes vivants à l'endroit où s'est produit la phosphatogenèse. Au cours de son dépôt, cette matière organique a subit diverses transformations qui ont abouti à trois catégories de composés : la première, le bitume soluble dans les solvants organiques. La deuxième catégorie, le kérogène, est insoluble dans les solvants organiques. Il est constitué de macromolécules organiques complexes et il est beaucoup plus abondant, en général, que le bitume. La troisième catégorie, les substances humiques qui sont solubles dans une solution basique.

La matière organique représente une teneur de 3 à $4 \%$ dans le sédiment phosphaté (phosphate noir de youssoufia) [1,2]. Divers travaux de recherche ont été orientés vers la détermination de la structure de la matière organique dans les sédiments [3-8]. 
Le présent travail concerne l'étude comparative des acides humiques et le kérogène qui sont extraits du phosphate de Youssoufia. Les analyses par spectroscopie infrarouge et la résonance magnétique nucléaire $d u{ }^{13} \mathrm{C} d u$ solide permettent de connaître la composition structurale de la matière organique. Le comportement thermique donne des informations sur les processus qui interviennent dans la dégradation lors de la calcination.

Dans un premier temps, nous avons extrait cette matière organique et nous l'avons isolée. Les acides humiques (A.H) et le concentré de kérogène (C.K) obtenus ont été étudiés dans le but de comparer leurs structures.

\section{Matériel et méthodes}

\section{2-1. Echantillon}

Le gisement de phosphate de Youssoufia est constitué de deux types de minerais. Un phosphate "I clair II contenant peu de matières organiques et le phosphate "I noir "I est, quant à lui, riche en matière organique qui représente un taux de $4 \%$. Sa situation géographique est à $100 \mathrm{~km}$ de Marrakech et à $230 \mathrm{~km}$ au sud de Casablanca, la zone de Youssoufia constitue la partie occidentale du gisement de Gantour. L'exploitation a commencé en 1931.

Les principaux éléments rencontrés dans l'échantillon à étudier, le phosphate noir de Youssoufia, sont répertories dans le Tableau 1 suivant :

Tableau 1 : Composition chimique du phosphate noir de Youssoufia.

\begin{tabular}{|c|c|}
\hline Composés & $\%$ \\
\hline $\mathrm{P}_{2} \mathrm{O}_{5}$ & 31,4 \\
\hline $\mathrm{CaO}$ & 50,2 \\
\hline Carbone organique & 2,0 \\
\hline Carbone minéral & 1,7 \\
\hline $\mathrm{SiO}_{2}$ & 1,4 \\
\hline $\mathrm{SO}_{3}$ & 1,6 \\
\hline $\mathrm{CaO} / \mathrm{P}_{2} \mathrm{O}_{5}$ & 1,5 \\
\hline Autres éléments & 10,2 \\
\hline
\end{tabular}

\section{2-2. Extraction du concentré de kérogène et des acides humiques}

La méthode utilisée consiste à récupérer le maximum de matière organique qui a été classée en trois grandes catégories: les lipides; les A.H et le C.K, [9], (Figure П). 
Après extraction des lipides libres par le mélange benzène/méthanol ( $1 / 2 \mathrm{vol} / \mathrm{vol})$ pendant 4 heures. Le solide restant, c'est à dire culot 1, est mélangé avec l'eau distillé puis acidifié par l'acide chlorhydrique tout en l'ajoutant par petites fractions jusqu'à $\mathrm{pH}=2$. Le mélange (solution et culot 1 ) est laissé à $\mathrm{pH}=2$ jusqu'à ce que le dégagement gazeux cesse. Le mélange est centrifugé à nouveau. Le solide est ensuite séché à une température de $80^{\circ} \mathrm{C}$ pendant 48 heures.

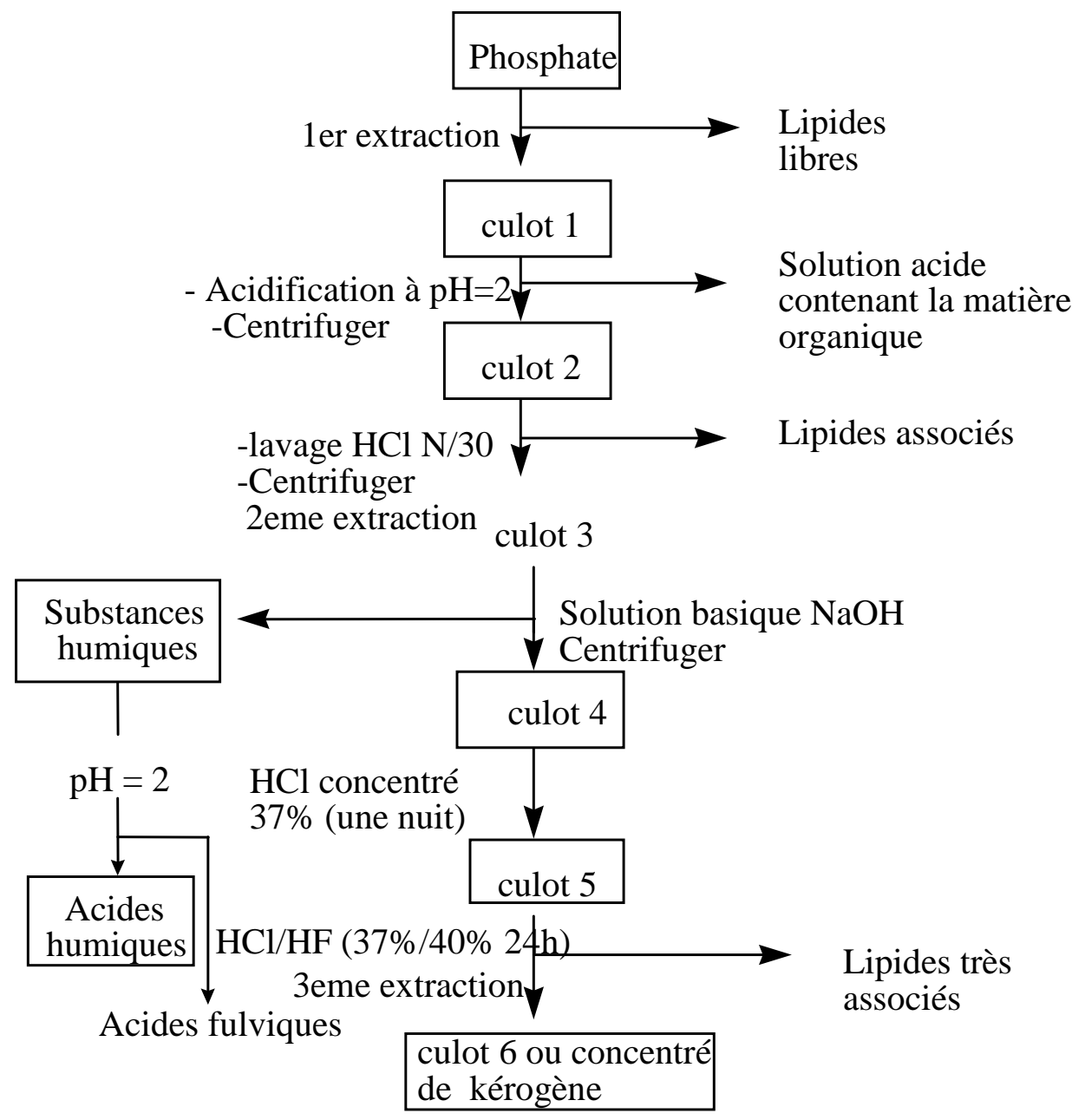

Figure 1 : Protocole d'extraction des acides humiques et du concentré de kérogène

Au niveau du culot 3, nous avons procédé à l'extraction des acides humiques par une solution basique $\mathrm{NaOH}(0,1 \mathrm{M})$ à raison de $10 \mathrm{~mL} / \mathrm{g}$ de solide. Dans un premier temps, le solide est mis dans un minimum d'eau. Le pH a été ajusté à 7 avec $\mathrm{NaOH}$ IM. Le mélange est agité pendant 4 heures puis laissé reposer 12 heures et centrifugé. Le solide 
récupéré est étuvé pendant une nuit à $80^{\circ} \mathrm{C}$. La solution basique est acidifiée jusqu'à $\mathrm{pH}=2$ et laissé reposer pendant 12 heures. Les A.H précipitent en milieu acide alors que les acides fulviques restent solubles. Les A.H sont obtenus après centrifugation pendant $15 \mathrm{mn}$ à 7000 tours $/ \mathrm{mn}$.

Le solide, exempt des acides humiques, est attaqué d'abord par l'acide $\mathrm{HCl}$ concentré $(37 \%)$ à température ambiante. Cette attaque permet d'éliminer la matière minérale et éviter la formation de la fluorine. Cette méthode diffère de celle utilisée habituellement pour les schistes. L'attaque directe par le mélange $\mathrm{HCl} / \mathrm{HF}$, sans élimination préalable du calcium apatitique, entrâne la formation de $\mathrm{CaF}_{2}$. Cet élément est insoluble dans une solution acide.

Au niveau du culot 6, nous avons éliminé tous les composés minéraux sauf les silicates. Leur dissolution est effectuée par $\mathrm{HF}$. Le résidu est mis en suspension dans une solution de $\mathrm{HCl} / \mathrm{HF}(1: 1)$ et est porté à $70^{\circ} \mathrm{C}$ pendant 8 heures. La température ne doit pas dépasser $70^{\circ} \mathrm{C}$ afin d'éviter une éventuelle oxydation de la matière organique. Les fluorures néoformés sont détruits par l'acide borique. Le résidu obtenu est le concentré de kérogène.

\section{2-3. Techniques spectroscopique}

La spectroscopie infrarouge à transformer de Fourrier (IR-TF) a été utilisée pour l'analyse des groupements fonctionnels existants dans le concentré de kérogène et les acides humiques. Une prise d'essai correspondant à $2 \%$ d'échantillon dans le KBr. L'appareil utilisé est un spectrophotomètre NICOLET 510.

Le spectre de la résonance magnétique nucléaire ${ }^{13} \mathrm{C}$ du solide (RMN ${ }^{13} \mathrm{C}$ solide) est obtenu avec l'appareil spectrophotomètre Bruker. L'ensemble de la procédure RMN ${ }^{13} \mathrm{C}$-rotation à l'angle magique-polarisation croisée-découplage constitue la technique ॥ CP/MAS $/{ }^{13} \mathrm{NMR}$ et permet d'obtenir des spectres à haute résolution. Le spectre est obtenu à $4,0 \mathrm{KHz}$ et les déplacements chimiques sont déterminés par rapport au tétraméthylsilane (TMS).

\section{2-4. Techniques thermogravimétrique et calorimétrique}

L'appareil utilisé est type STA 409 EP. II permet de suivre la perte de masse (ATG) et le flux thermique (DSC) en fonction de la température sous air dont le débit est fixé à 50 $\mathrm{ml} / \mathrm{mn}$. Le creuset est en platine. Les essais ont été effectués en régime dynamique jusqu'à $800^{\circ} \mathrm{C}$, à vitesse de chauffe constante égale à $5^{\circ} \mathrm{C} / \mathrm{mn}$.

\section{2-5. Pyrolyse}

Le dispositif expérimental qui a servi à l'étude de la cinétique de formation des gaz comporte un réacteur à lit fixe en régime dynamique sous atmosphère inerte (Ar). Le four 
est commandé par un programmateur linéaire de température qui peut faire varier la vitesse de chauffage de 1 à $40^{\circ} \mathrm{C} / \mathrm{mn}$. Le réacteur est connecté, à la sortie, à un piège à $0^{\circ} \mathrm{C}$ empêcher les matières organiques liquides d'atteindre le système de détection. L'analyse des gaz est effectué par chromatographie en phase gazeuse $[1,10]$.

\section{Résultats et discussion}

\section{3-1. Caractérisation par spectroscopie infrarouge}

Sur la Figure 2, nous représentons les spectres d'absorptions I.R du C.K (courbe a) et des A.H (courbe b). Ces spectres permettent d'identifier les différentes bandes d'absorptions et les principaux groupements [11]:

- Les deux spectres présentent une différence au niveau des bandes d'absorptions dans la région $3500-2700 \mathrm{~cm}^{-1}$. Le spectre (a) possède deux bandes larges à 2925 et $2854 \mathrm{~cm}^{-1}$ attribuées généralement aux groupements alkyls. Ces deux bandes sont de très faible intensité sur le spectre (b). Sur le spectre (a), nous constatons l'apparition de deux bandes situées respectivement à 1447 et $1377 \mathrm{~cm}^{-1}$ dues à des vibrations de déformation de - $\mathrm{CH}_{2}$ et - $\mathrm{CH}_{3}$. Ceci montre que le $\mathrm{C} . \mathrm{K}$ présente une prédominance marqué des composés aliphatiques.

Sur les deux spectres ( $a$ et $b$ ), ils existent des bandes supplémentaires comprises entre 1800 et $1500 \mathrm{~cm}^{-1}$ :

* absorption vers $1612 \mathrm{~cm}^{-1}$ attribuée à la vibration $C=C$ du cycle aromatique (spectre $b$ ). * les groupements $C=0$ appartenant à la fonction ester ou acide possèdent une vibration de valence aux environs du nombre d'onde égal à $1636 \mathrm{~cm}^{-1}$ (spectre a). L'absorption à 1706 ou $1707 \mathrm{~cm}^{-1}$ est due à la vibration de valence $C=0$ des groupements carboxyliques acides saturés. En plus des bandes situées entre $1100-1300 \mathrm{~cm}^{-1}$ semblent être liées à la vibration de valence de la liaison $\mathrm{C}-0$ des fonctions esters ou acides.

- Entre 1200 et $900 \mathrm{~cm}^{-1}$ : un massif mal défini contenant des bandes correspondant à des vibrations $\mathrm{C}=\mathrm{C}$ aromatiques; $\mathrm{C}-\mathrm{C} ; \mathrm{C}-\mathrm{O}$ et $\mathrm{O}-\mathrm{H}$ (phénols et alcools).

- Entre 900 et $700 \mathrm{~cm}^{-1}$ : vibration de déformation hors du plan des - $\mathrm{CH}$ aromatiques. 


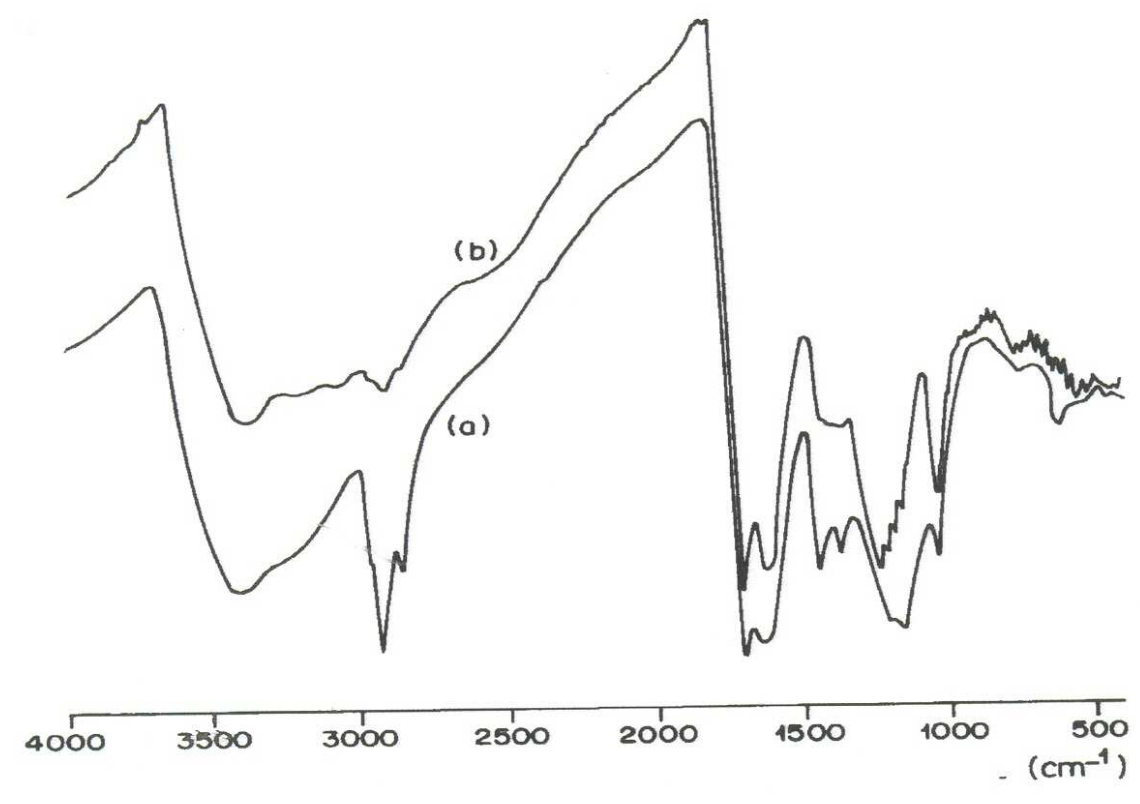

Figure 2 : Spectre infrarouge des deux échantillons

(a) concentré de kérogène

(b) acides humiques

\section{3-2. Caractérisation par spectroscopie R.M.N ${ }^{13} \mathrm{C}$ du solide}

Les deux spectres a et b de la Figure 3 représentent les résultats de la R.M.N ${ }^{13} \mathrm{C}$ respectivement du C.K et des A.H. Ils comportent quatre bandes caractéristiques [12,13]:

- Une bande compris entre 0 et $50 \mathrm{ppm}$ possédant un maximum aux environs de $30 \mathrm{ppm}$ attribuée à des composés aliphatiques de structure polyethyléniques. La présence de groupements methyl $\left(-\mathrm{CH}_{3}\right)$ est révélée par un pic caractéristique à $15 \mathrm{ppm}$. Cette bande est observée uniquement sur le spectre (a) correspondant au C.K.

- Entre 60 et 110 ppm, apparaît la bande caractéristique des résonances des composés du type $(C-0)$. Sur le spectre $(a)$, cette bande existe sous forme d'un massif indiquant la présence de plusieurs forme de liaison $\mathrm{C}-0$.

- La région située entre 110 ef 160 ppm correspond à la résonance des carbones hybridés en $\mathrm{sp}^{2}(\mathrm{C}=\mathrm{C})$. Ce sont des carbones aromatiques. Ces bandes ont un maximum aux environs de $130-135 \mathrm{ppm}$ et correspondent à des carbones aromatiques alkylés. Les carbones aromatiques oxygénés apparaissent vers 154 ppm.

- Une bande apparaît dans la région 160 - 200 ppm, son sommet est situé à 180 $\mathrm{ppm}$. Cette bande est attribuable à la liaison $c=0$ des composés esters, des groupements carboxyliques et aussi des composés amides. 
Le calcul du facteur d'aromaticité du C.K et des A.H a conduit à des valeurs égales à 0,29 et 0,545 .

D'après ces résultats spectroscopiques, nous constatons que:

- le C.K est de nature aliphatique,

- les A.H sont de natures aromatiques.

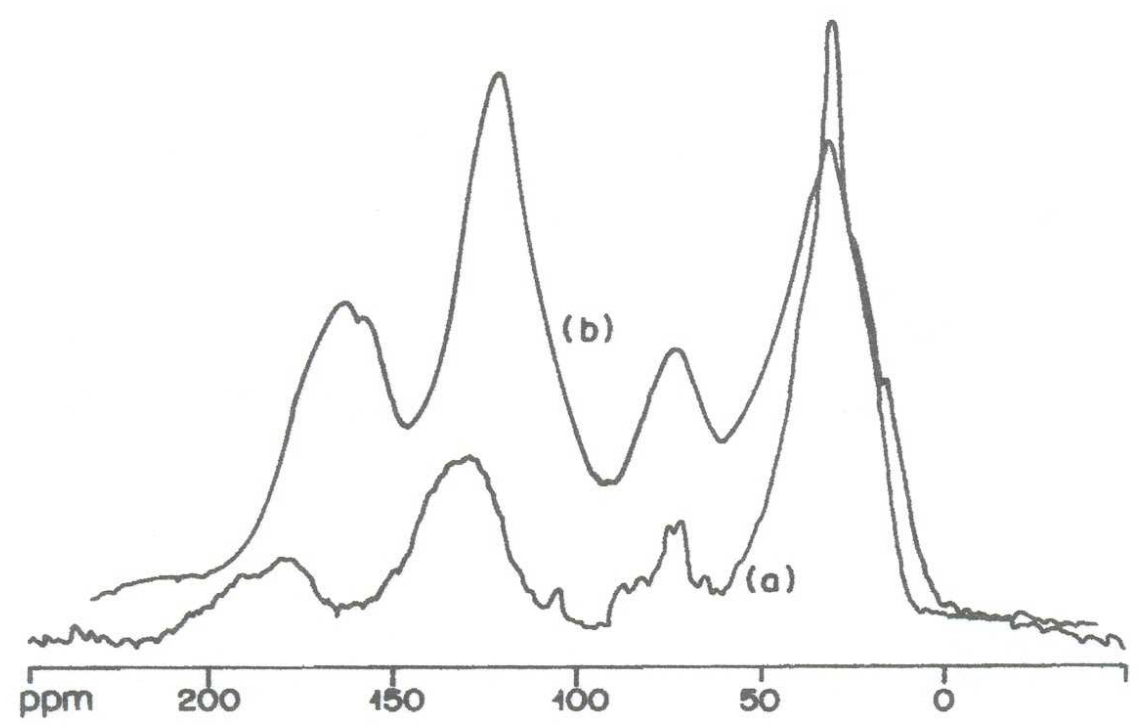

Figure $3: R M N{ }^{13} \mathrm{C} d u$ solide des deux échantillons

(a) concentré du kérogène

(b) acides humiques

\section{3-3. Analyse thermogravimétrique et calorimétrique}

Les courbes thermogravimétriques (ATG) des deux échantillons C.K et A.H sont représentées respectivement sur les deux Figures 4 et 5 . La perte de masse s'effectue dans un intervalle de température allant de $40^{\circ} \mathrm{C}$ jusqu'aux environs $580^{\circ} \mathrm{C}$. Sur les deux figures d'ATG, on constate que:

- il y a une perte qui se produit entre 40 et $100^{\circ} \mathrm{C}$. Elle est due principalement au départ de l'eau.

- il y a une autre perte entre $100^{\circ} \mathrm{C}$ et $580^{\circ} \mathrm{C}$. La courbe d'ATG de la figure 4 comporte plusieurs accidents correspondant à des pertes de masses différentes. La perte totale est équivalente à $85 \%$. Par contre, la perte de masse des A.H sous air s'effectue en une seule étape qui représente $90 \%$. 
Pour l'analyse en DSC du C.K, (Figure 4), on constate l'apparition de plusieurs pics exothermiques dans l'intervalle de température $200-500^{\circ} \mathrm{C}$. Le premier pic est le plus intense et correspond au début du processus de l'oxydation de la matière organique. Dans l'intervalle $200-340^{\circ} \mathrm{C}$ commence le dégagement de $\mathrm{CO}_{2}$ issu des composés esters ou acides suivi de l'oxydation des carbones de ces mêmes composés. Au dessus de $340^{\circ} \mathrm{C}$, on observe la présence de trois principaux pics correspondant au deuxième type de matière organique. II est difficile d'attribuer à chaque pic une réaction bien définie. Néanmoins, on peut envisager l'existence de deux types de matière organique. Le premier type commence à se manifester à des températures inférieures à $350^{\circ} \mathrm{C}$. La dégradation du deuxième type commence à partir de $350^{\circ} \mathrm{C}$ jusqu'à $500^{\circ} \mathrm{C}$. II semble que ce dernier type de matière organique est constitué de plusieurs composés différents.

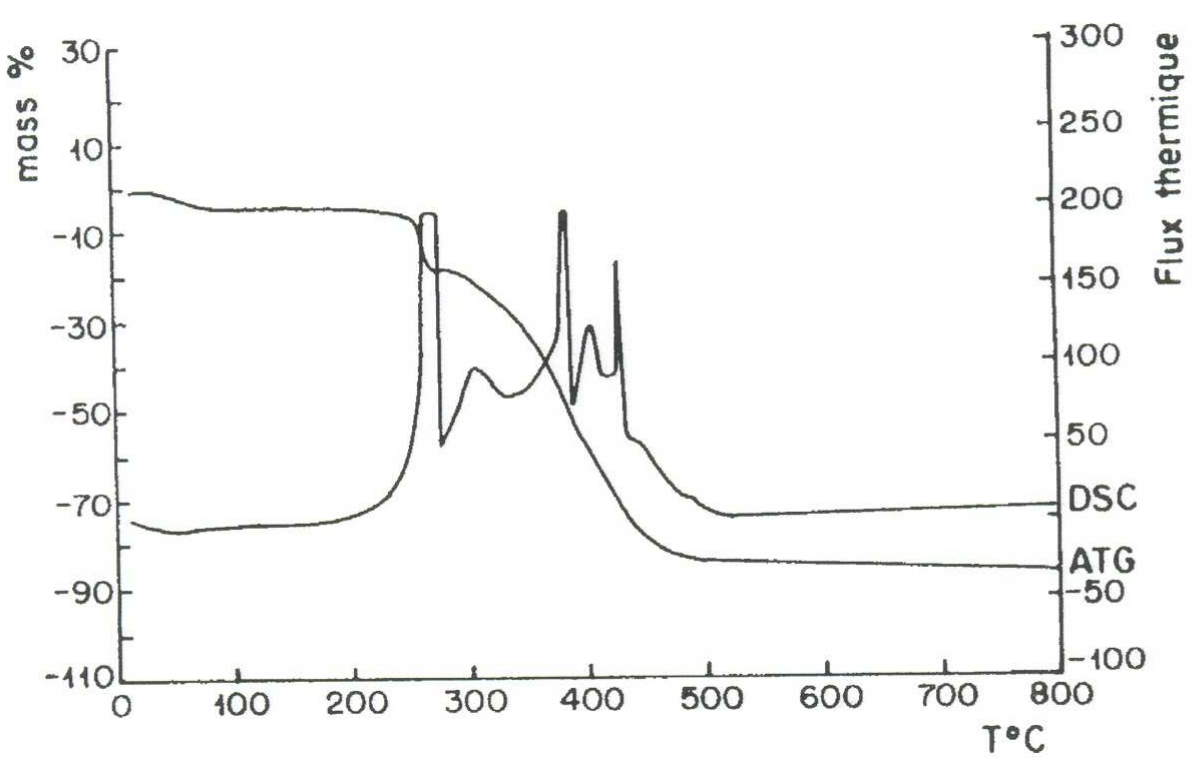

Figure 4 : ATG et DSC du concentré du kérogène sous atmosphère oxydante (air)

Les A.H présentent un seul pic dont le maximum est situé aux environs de $400^{\circ} \mathrm{C}$ (Figure 5). Ce pic n'est probablement pas dô à un composé bien défini car il ne possède pas une allure gaussienne. On pense que les $A . H$ sont constitués d'un seul type de composé organique. 


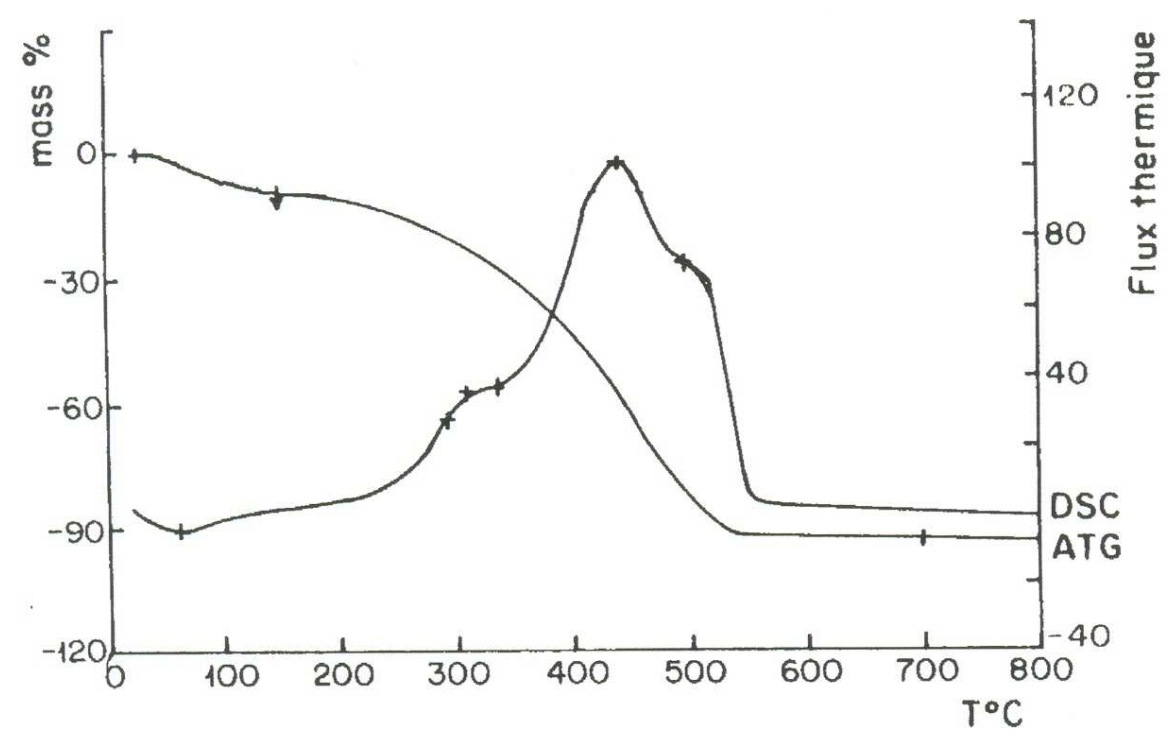

Figure 5 : ATG et DSC des acides humiques sous atmosphère oxydante (air)

\section{3-4.- Analyse des gaz}

Sur la Figure 6, nous avons représenté les courbes donnant la vitesse de dégagement des principaux gaz: $\mathrm{CO}_{2}, \mathrm{H}_{2}$, et $\mathrm{CH}_{4}$ en atmosphère inerte du $\mathrm{C} . \mathrm{K}$.

- Le premier dégagement observé est celui du $\mathrm{CO}_{2}$. Sa production s'effectue en deux vagues dans l'intervalle de température allant de 150 jusqu'aux environs $550^{\circ} \mathrm{C}$. Les deux vagues indiquent que la formation du $\mathrm{CO}_{2}$ provient des deux sources différentes. La première vague, située à $300^{\circ} \mathrm{C}$, est due à la décomposition des acides carboxyliques existant dans la matière organique du C.K. La seconde vague, moins importante, apparaît aux environs de $420^{\circ} \mathrm{C}$. Elle est liée à la dégradation des fonctions esters.

- L'apparition de $\mathrm{CH}_{4}$ commence à partir de $320^{\circ} \mathrm{C}$ jusqu'à $650^{\circ} \mathrm{C}$. Son dégagement $s^{\prime}$ effectue en une seule vague. II provient de la dégradation directe de la matière organique.

- L'hydrogène apparaît dans l'intervalle de 400 à $850^{\circ} \mathrm{C}$ en une seule vague. La courbe montre que le maximum de production est situé aux environs de $680^{\circ} \mathrm{C}$. 


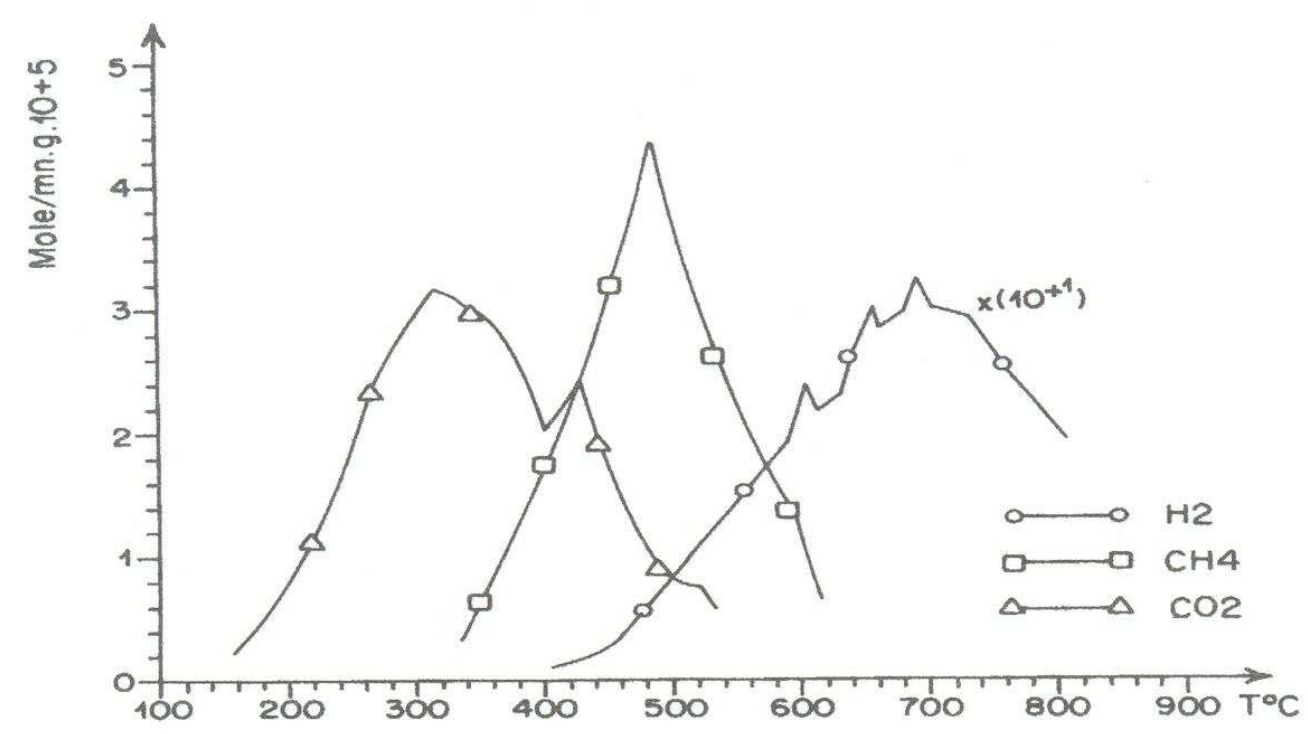

Figure 6 : Evolution des principaux gaz dégagés du concentré du kérogène

Sur la Figure 7 , nous avons rassemblé la séquence de dégagement des principaux gaz des A.H:

- Le $\mathrm{CO}_{2}$ est produit dans l'intervalle de température $(150 ; 650)$. Il se dégage en une seule vague dont le maximum est situé à $300^{\circ} \mathrm{C}$. L'origine de production de $\mathrm{CO}_{2}$ est due principalement à la décomposition des composés acides.

- Le méthane se dégage en une seule vague presque symétrique dans un intervalle de température allant de $320^{\circ} \mathrm{C}$ jusqu'à $650^{\circ} \mathrm{C}$.

- L'hydrogène ne commence à apparaître que lorsque le méthane a atteint son maximum de dégagement. Ceci indique que l'hydrogène $\left(H^{\circ}\right)$ formé réagit dans un premier temps avec les methyls $\left(-\mathrm{CH}_{3}^{\circ}\right)$ pour former le méthane. La production d'hydrogène s'effectue en une seule vague dont le maximum de production est situé aux environs de $740^{\circ} \mathrm{C}$. 


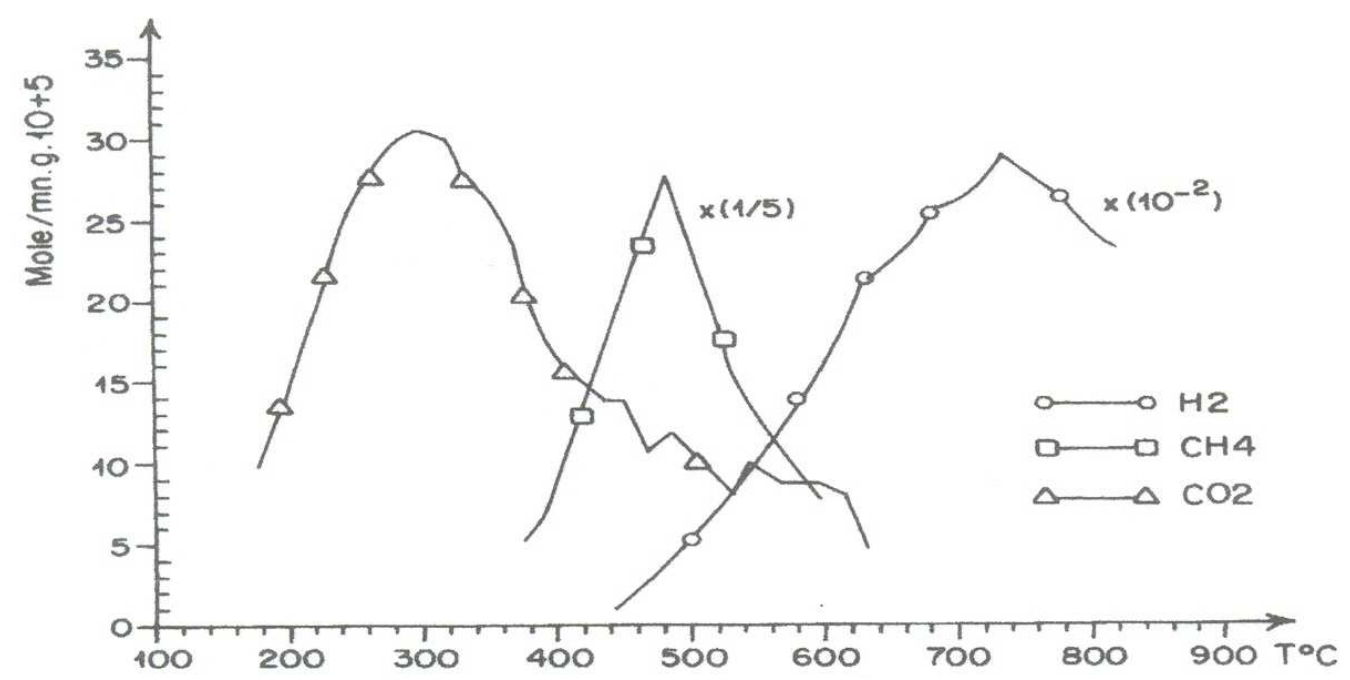

Figure 7 : Evolution des principaux gaz dégagés des acides humiques

\section{Conclusion}

La composition du concentré du kérogène présente une prédominance des composés aliphatiques, alors que les acides humiques n'en contient que très peu. II existe plus des composés aromatiques dans les acides humiques que dans le concentré du kérogène et il est probable que les fonctions esters n'existent que dans le concentré du kérogène. Le $\mathrm{CO}_{2}$ dégagé des acides humiques est très important comparé à celui dégagé du concentré du kérogène La température correspondant au maximum de production de $\mathrm{CO}_{2}$ des acides humiques est la même que celle du premier pic de la courbe du $\mathrm{CO}_{2}$ issus du concentré du kérogène.

L'allure de la courbe cinétique de dégagement de $\mathrm{CH}_{4}$ des acides humiques est identique à celle du concentré du kérogène La production d'hydrogène par le concentré du kérogène est très importante comparée à celle des acides humiques.

\section{Références}

[1] - M. Khaddor, Thèse, Université Mohammed V-Agdal, Rabat (1988)

[2] - A. Bouhaouss, Thèse, Université Mohammed V-Agdal, Rabat (1980)

[3] - V. Bruan, M. Halim, M. Ziyad, C. Largeau and A. Amblès, Journal of Analytical and Applied Pyrolysis, Vol 61, Issues 1-2 (2001) 165-179 
[4] - M. Ziyad, M. Khaddor et M. Halim, Fuel, Vol 72, Number 5 (1993) 655-659

[5] - M. Khaddor, M. Ziyad, M. Halim, J. Joffre et A. Amblès, Fuel, Vol 76, Number 14/15 (1997) 1395-1400

[6] - X. De Las Heras, J. 0. Gumalt, J. Albaiges, R. Julia et P. Anadon, Org. Geochem, Vol $14, N^{\circ} 6$ (1989) 667-677

[7] - V. Gobé, L. Lemée et A. Amblès, Org Geochem Vol 31 (2000) 400-419

[8] - B. Gillaizeau, S. Derenne, C. Largeau, C. Berkaloff et B. Rousseau, Org. Geochem, Vol 24, Nº/7 (1996) 671-679

[9] - G. Calderoni et M. Schnitzer, Org. Geochem, Vol 5, Nº 4 (1984) 203-209

[10] - M. Khaddor, M. Ziyad, J. Joffre et A. Amblès, Chemical Geology Vol 186 (2002) 17-30

[11] - P.G. Roschet, P.L. Robin et G. Nicaise, "Kerogen (insoluble Organic Matter from Sedimentary Rocks)", Ed. bernard durant, Institut français du pétrole (1980)

[12]- C. Largeau, S. Derenne, E. Casadevall and N. Sellier, Org. Geochem, Vol 10, (1986) 1023-1032

[13]- F. Martin, G. Almendros, F. J. González-Vila and T. Verdejo, Journal of Analytical and Applied Pyrolysis, Vol 61, Issues 1-2 (2001) 133-145 